\title{
The effect of nanoparticle chain formation on dielectric anisotropy of nematic composites
}

\author{
M.A. Osipov ${ }^{1}$ and M.V. Gorkunov ${ }^{2}$ \\ ${ }^{1}$ Department of Mathematics, University of Strathclyde, Glasgow G1 1XH, Scotland, UK \\ ${ }^{2}$ Shubnikov Institute of Crystallography of Russian Academy of Sciences, Moscow 119333, Russia
}

\begin{abstract}
A general theory of the dielectric constant of nematic liquid crystal mixtures is presented including the particular case of nematics doped with polar nanoparticles. The results are used to estimate the contribution of chains of polar nanoparticles to the static dielectric anisotropy and birefringence of the nematic composite taking into account contributions from chains of different lengths. The dependence of the dielectric anisotropy on the dipolar interaction strength is considered in detail and it is shown that formation of polar chains of nano-particles enables one to explain a significant increase of the dielectric constant of the composite as observed experimentally.
\end{abstract}

PACS numbers: 64.70.mf, 61.30.Cz, 42.70.Df

\section{INTRODUCTION}

There has been significant recent interest in novel liquid crystal (LC) nano-composites, i.e., LCs doped with metal, dielectric or semiconductor nanoparticles (NPs) of scales of 2-10 nm (i.e. close to the size of typical mesogenic molecules). It has been shown that doping of a nematic LC with even a small NP volume concentration can affect almost all important nematic phase properties, e.g. decrease the threshold and switching voltages as well as switching times of LC displays (see, for example, Refs. 15). Suspensions of different NPs in various nematic LCs have been investigated by many authors. In particular, nematics doped with ferroelectric NPs enhance dielectric and optical anisotropy, increase the electro-optic response $[6,7]$ and improve the photorefractive properties of LC composites [8]. Nematic suspensions of paraand ferromagnetic particles are promising candidates for magnetically tunable structures, and doping of ferroelectric LCs with metal and silica NPs can improve spontaneous polarization and dielectric permittivity, as well as decrease the switching times $[9,10]$. Metal NPs have been also used to widen the temperature range of $\mathrm{LC}$ blue phases [11], which are important for applications, and enhance random lasing in the dye-doped LC medium [12].

It has also been shown experimentally (see, for example, Refs. 9 and 13) that the dielectric anisotropy of nematic LCs doped with strongly polar (ferroelectric) NPs is dramatically increased. Indeed, a very small molar fraction of ferroelectric NPs (of the order of $10^{-3}$ ) accounts for a contribution to the relative dielectric anisotropy of the order of $5-6$, i.e., comparable with the anisotropy of the nematic host. Preliminary estimates indicate that the increase is too strong to be explained without taking into account possible aggregation of NPs and formation of polar chains. There exists some experimental evidence that quantum dots may also form long chains in nematic LCs [14] even though such NPs are nonpolar. Aggregates of NPs in general, and polar chains in particular, would be expected to modify all major properties of nematic nano-composites, includ- ing their dielectric and optical properties. Nematic LCs with polar chains should also be very sensitive to external electric fields which may be used for alignment and switching at very low applied voltage.

Aggregation of NPs in the nematic phase may occur if the inter-particle interaction potential is not strong enough to induce demixing but is still much stronger than the interaction between mesogenic molecules. Strongly anisotropic interaction between NPs, including, in particular, the dipole-dipole one, will lead to the formation of polar chains. It has been shown [13] that the equilibrium chain length strongly depends on the contact interaction potential normalized by the temperature. Long chains of NPs may occur only if the contact interaction is of the order of $10 k_{B} T$ [15] which is satisfied, for example, for ferroelectric NPs $[9,13]$. Such long polar chains should make a significant contribution to the dielectric anisotropy of nematic composites.

The theory of LCs doped with NPs is still at a rudimentary stage. Lopatina and Selinger [10] showed phenomenologically that dipole-dipole interactions between ferroelectric NPs can significantly increase the isotropicnematic (I-N) phase transition temperature. Gorkunov and Osipov [16] used molecular theory to make a detailed analysis of the effect of both anisotropic and isotropic NPs on the properties of the I-N transition. The theory developed in Ref. 16 has been later used to describe the effect of external electric field on nematic nanocomposites [17]. A molecular theory of nematic LCs doped with spherical NPs has also been developed in Ref. 18. Very recently, a detailed theory of the phase separation effects in nematic LCs doped with isotropic NPs has been presented by the authors [19]. In this paper, we develop a general theory of the dielectric and optical properties of polar nematic mixtures and estimate the contribution of polar NPs and chains of NPs to the nano-composite dielectric anisotropy and birefringence.

The paper is arranged as follows. In Section II a general molecular-statistical theory of the dielectric susceptibility of many component nematic mixtures is presented taking into consideration separately the high and 
low frequency limits. In Section III, a contribution of chains of strongly polar ferroelectric NPs to the dielectric anisotropy of the composite nematic phase is calculated taking into account statistics of chains of various length. The numerical and analytical results are presented to illustrate the dependence of the dielectric anisotropy on the concentration of NPs and on the strength of their dipole-dipole interaction. Theoretical results are then used to explain the existing experimental data. Finally, Section IV contains a discussion.

\section{GENERAL THEORY OF THE DIELECTRIC SUSCEPTIBILITY OF MANY COMPONENT NEMATICS}

\section{A. Microscopic polarization and the electric field}

The microscopic polarization $\mathbf{P}^{M}$ of a many component LC consists of the contributions from all the fluctuating molecular and particle dipoles:

$$
\mathbf{P}^{M}(\mathbf{r}, t)=\sum_{i, \alpha} \mathbf{p}_{i, \alpha} \delta\left(\mathbf{r}-\mathbf{r}_{i}\right),
$$

where $\mathbf{p}_{i, \alpha}$ is the total dipole of the $i$-th molecule/particle of the component $\alpha$ located at the point $\mathbf{r}_{i}$. In the general case, the dipole $\mathbf{p}_{i, \alpha}$ is the sum of the permanent molecular dipole $\mathbf{p}_{i, \alpha}^{0}$ and the dipole $\mathbf{p}_{i, \alpha}^{i n}$ induced by the local electric field.

The microscopic polarization is related to the microscopic electric field $\mathbf{E}^{M}$ by the following well known equation:

$$
\text { curl curl| } \mathbf{E}^{M}+\frac{1}{c^{2}} \frac{\partial^{2} \mathbf{E}^{M}}{\partial t^{2}}=-\frac{1}{c^{2}} \frac{\partial^{2} \mathbf{P}^{M}}{\partial t^{2}} .
$$

The general solution of Eq. (2) can be expressed in the operator form [20]:

$$
\mathbf{E}^{M}=\mathbf{E}_{0}+\int \hat{F}\left(\mathbf{r}-\mathbf{r}^{\prime}, \omega\right) \mathbf{P}^{M}(\mathbf{r}, \omega) d \mathbf{r}^{\prime},
$$

where $\mathbf{E}_{0}$ is an external (homogeneous) electric field and the operator kernel $\hat{F}\left(\mathbf{r}-\mathbf{r}^{\prime}, \omega\right)$ reads [20]:

$$
\begin{aligned}
& \hat{F}(\mathbf{R}, \omega)=\frac{4 \pi}{3} \delta(\mathbf{R})- \\
& {\left[\left(1+\frac{i \omega R}{c}-\frac{\omega^{2} R^{2}}{3 c^{2}}\right)(\hat{I}-3 \mathbf{u} \otimes \mathbf{u})-\frac{2 \omega^{2} R^{2}}{3 c^{2}}\right] \times} \\
& e^{i \omega R / c} R^{-3},
\end{aligned}
$$

where $\mathbf{u}=\mathbf{R} / R$.

One notes that in practice the distance $\mathbf{R}=\mathbf{r}-\mathbf{r}^{\prime}$ is limited by the correlation radius $\xi$ which is always smaller then the wavelength of light $\lambda$. Thus $\omega R / c \ll 1$ and then the kernel $\hat{F}(\mathbf{R}, \omega)$ is reduced to the simple form of the dipole-dipole propagator:

$$
\hat{F}(\mathbf{R}, \omega)=\frac{4 \pi}{3} \delta(\mathbf{R})+(3 \mathbf{u} \otimes \mathbf{u}-\hat{I}) R^{-3} .
$$

\section{B. High frequency permittivity of a nematic composite}

At sufficiently high (optical) frequencies, the polarization is mainly determined by induced dipoles created by the electric field. Orientational fluctuations of permanent dipoles make a minor contribution because the characteristic times of such fluctuations are much larger than the inverse optical frequency [21]. Then the molecular dipole in Eq. (1) can be expressed approximately as

$$
\mathbf{p}_{i, \alpha}=\hat{\beta}_{\alpha}\left(\theta_{i}, \omega\right) \mathbf{E}^{M}\left(\mathbf{r}_{i}, \omega\right),
$$

where $\hat{\beta}_{\alpha}\left(\theta_{i}, \omega\right)$ is the polarizability of the molecule $i$ of the component $\alpha$, the variable $\theta_{i}$ describes the molecule orientation, and the field $\mathbf{E}^{M}\left(\mathbf{r}_{i}, \omega\right)$ acting on the molecule $i$, is a sum of the external field $\mathbf{E}_{0}\left(\mathbf{r}_{i}, \omega\right)$ and the electric field created by the dipoles induced in all other molecules:

$$
\mathbf{E}^{M}\left(\mathbf{r}_{i}, \omega\right)=\mathbf{E}_{0}\left(\mathbf{r}_{i}, \omega\right)+\sum_{j, \alpha} \hat{F}\left(\mathbf{r}_{i}-\mathbf{r}_{j}, \omega\right) \mathbf{p}_{j, \alpha} .
$$

These equations can be used to obtain the closed equation for the microscopic field:

$$
\mathbf{E}^{M}(\mathbf{r}, \omega)=\mathbf{E}_{0}-\int \hat{H}\left(\mathbf{r}-\mathbf{r}^{\prime}, \omega\right) \hat{\gamma}(\omega) \mathbf{E}^{M}\left(\mathbf{r}^{\prime}, \omega\right) d \mathbf{r}^{\prime},
$$

where

$$
\hat{\gamma}(\omega)=\sum_{\alpha} \int \hat{\beta}_{\alpha}(\theta, \omega) \rho_{\alpha}(\theta, \mathbf{r}) d \theta
$$

is a microscopic polarizability, and $\rho_{\alpha}$ is the microscopic number density of the molecules of type $\alpha$ :

$$
\rho_{\alpha}(\theta, \mathbf{r})=\sum_{i} \delta\left(\mathbf{r}-\mathbf{r}_{i}\right) \delta\left(\theta-\theta_{i}\right)
$$

Here the operator kernel $\hat{H}=\hat{F}$ when $\left|\mathbf{r}-\mathbf{r}^{\prime}\right|>D$ and $\hat{H}=0$ when $\left|\mathbf{r}-\mathbf{r}^{\prime}\right|<D$, where $D$ is the molecular diameter.

The macroscopic polarization $\mathbf{P}$ in the media is the statistical average of the microscopic polarization $\mathbf{P}=$ $\left\langle\mathbf{P}^{M}\right\rangle$, where $\langle\ldots\rangle$ denotes the ensemble average. The polarization can also be expressed as $\mathbf{P}=\hat{\chi} \mathbf{E}$, where $\mathbf{E}=\left\langle\mathbf{E}^{M}\right\rangle$ is the macroscopic electric field and $\hat{\chi}$ is the dielectric susceptibility tensor of the medium.

Writing Eq. (8) in the operator form as $\mathbf{E}^{M}=\mathbf{E}_{0}$ $\hat{\mathcal{H}} \hat{\gamma} \mathbf{E}^{M}$, one can readily express the microscopic field by the external electric field $\mathbf{E}_{0}$ :

$$
\mathbf{E}^{M}=(1+\hat{\mathcal{H}} \hat{\gamma})^{-1} \mathbf{E}_{0},
$$

which determines the microscopic polarization as

$$
\mathbf{P}^{M}=\hat{\gamma}(1+\hat{\mathcal{H}} \hat{\gamma})^{-1} \mathbf{E}_{0}
$$

and yields the average polarization

$$
\mathbf{P}=\left\langle\hat{\gamma}(1+\hat{\mathcal{H}} \hat{\gamma})^{-1}\right\rangle \mathbf{E}_{0} .
$$


A relationship between the external field $\mathbf{E}_{0}$ and the average field $\mathbf{E}$ can be obtained using Eqs. (3,5,12). Substituting Eq. (5) into Eq. (3) one obtains:

$$
\mathbf{E}^{M}=\mathbf{E}_{0}-\frac{4 \pi}{3} \mathbf{P}^{M}-\int \hat{H}\left(\mathbf{r}-\mathbf{r}^{\prime}, \omega\right) \mathbf{P}^{M}(\mathbf{r}, \omega) d \mathbf{r}^{\prime}
$$

Expressing $\mathbf{P}^{M}$ in terms of $\mathbf{E}_{0}$ (using Eq. (12)) in the third term in Eq. (14) and combining the two terms proportional to $\mathbf{E}_{0}$, one obtains:

$$
\mathbf{E}^{M}+\frac{4 \pi}{3} \mathbf{P}^{M}=(1+\hat{\mathcal{H}} \hat{\gamma})^{-1} \mathbf{E}_{0}
$$

where we have taken into account that $\hat{I}-\hat{\mathcal{H}} \hat{\gamma}(1+$ $\hat{\mathcal{H}} \hat{\gamma})^{-1}=(1+\hat{\mathcal{H}} \hat{\gamma})^{-1}$.

Upon averaging, Eq. (15) yields:

$$
\mathbf{E}_{0}=\left\langle(1+\hat{\mathcal{H}} \hat{\gamma})^{-1}\right\rangle^{-1}\left(\mathbf{E}+\frac{4 \pi}{3} \mathbf{P}\right)
$$

Finally, substituting Eq. (16) into Eq. (13) one obtains the following relationship between the average polarization and the average electric field in the nematic medium:

$$
\mathbf{P}=\left\langle\hat{\gamma}(1+\hat{\mathcal{H}} \hat{\gamma})^{-1}\right\rangle\left\langle(1+\hat{\mathcal{H}} \hat{\gamma})^{-1}\right\rangle^{-1}\left(\mathbf{E}+\frac{4 \pi}{3} \mathbf{P}\right)
$$

which yields for the permittivity tensor:

$$
(\hat{\varepsilon}-1)(\hat{\varepsilon}+2)^{-1}=\frac{4 \pi}{3} \hat{\alpha},
$$

where

$$
\hat{\alpha}=\left\langle\hat{\gamma}(1+\hat{\mathcal{H}} \hat{\gamma})^{-1}\right\rangle\left\langle(1+\hat{\mathcal{H}} \hat{\gamma})^{-1}\right\rangle^{-1}
$$

Thus the general equation for the high frequency permittivity of the nematic doped with NPs has the form of the generalized Clausius-Mossotti equation in which the average polarizability of a single molecule is replaced by the effective renormalized polarizability that depends both on intermolecular interactions and correlations and on interactions and correlations between NPs and neighboring mesogenic molecules. One notes also that Eq. (18) is a generalization of the corresponding equation for isotropic fluids.

In Eq. (19), the fluctuating quantity is the weighted microscopic polarizability $\hat{\gamma}$ which depends on the orientation and position of both mesogenic molecules and the NPs. The renormalized polarizability $\hat{\alpha}$ can be expanded in powers of the polarizability fluctuation $\Delta \gamma=\gamma-\langle\gamma\rangle$ where $\langle\gamma\rangle$ is the average polarizability:

$$
\hat{\alpha}=\langle\gamma\rangle-\langle\Delta \gamma \hat{K} \Delta \gamma\rangle+\ldots
$$

where $\hat{\mathcal{K}}=(1+\hat{\mathcal{H}}\langle\hat{\gamma}\rangle)^{-1} \hat{\mathcal{H}}$

Taking into account that $\hat{\gamma}$ is a weighted sum of polarizabilities of all components of the mixture (including mesogenic molecules, NPs and chains of NPs of different length) given by Eq. (9), one obtains:

$$
\hat{\alpha}=\sum_{\alpha}\left\langle\hat{\beta}_{\alpha}\right\rangle \rho_{\alpha}-\sum_{\alpha, \beta} \hat{\lambda}_{\alpha, \beta} \rho_{\alpha} \rho_{\beta}+\ldots,
$$

where $\left\langle\hat{\beta}_{\alpha}\right\rangle$ is the average polarizability of the component $\alpha$ and

$$
\hat{\lambda}_{\alpha, \beta}=\int g_{\alpha, \beta}\left(\mathbf{R}, \theta, \theta^{\prime}\right) \hat{\beta}_{\alpha}(\theta) \hat{K}(\mathbf{R}, \omega) \hat{\beta}_{\beta}\left(\theta^{\prime}\right) d \mathbf{R} d \theta d \theta^{\prime} .
$$

Here $g_{\alpha, \beta}\left(\mathbf{R}, \theta, \theta^{\prime}\right)$ are the pair correlation functions between the molecules of the components $\alpha$ and $\beta$, and $\hat{K}(\mathbf{R}, \omega)$ is the kernel of the operator $\hat{\mathcal{K}}$. The higher order terms in Eqs. (20) and (21) depend on higher order correlation functions.

Relatively simple explicit expressions for the permittivity can be obtained in the molecular field approximation, when one neglects the correlation corrections $\hat{\lambda}_{\alpha, \beta}$. In this case, Eq. (18) can be written in the following form assuming that the composite nematic phase contains mesogenic molecules, NPs and chains of NPs of various lengths $n$ :

$$
\begin{aligned}
& (\hat{\epsilon}-1)(\hat{\epsilon}+2)^{-1}= \\
& \frac{4 \pi}{3}\left(\left\langle\hat{\beta}_{m}\right\rangle \rho_{m}+\left\langle\hat{\beta}_{n p}\right\rangle \rho_{n p}+\sum_{n=2}^{\infty}\left\langle\hat{\beta}_{n}\right\rangle \rho_{n}\right)
\end{aligned}
$$

where $\left\langle\hat{\beta}_{m}\right\rangle,\left\langle\hat{\beta}_{n p}\right\rangle$ and $\left\langle\hat{\beta}_{n}\right\rangle$ are the average polarizabilities of mesogenic molecules, NPs and chains of NPs of length $n$, respectively, and $\rho_{m}, \rho_{n p}$ and $\rho_{n}$ are the corresponding number densities.

In a uniaxial nematic composite, all the components are distributed uniaxially around the same nematic director vector $\mathbf{n}$. Introducing the long axes of the molecules $\mathbf{a}_{m}$ and the unit vectors of the chain directions $\mathbf{a}_{n}$, one can write their momentary polarizabilities as $\hat{\beta}_{\alpha}=\beta_{\alpha \perp} \hat{I}+\Delta \beta_{\alpha} \mathbf{a}_{\alpha} \otimes \mathbf{a}_{\alpha}$, express the averages $\left\langle\mathbf{a}_{\alpha} \otimes \mathbf{a}_{\alpha}\right\rangle=\hat{I}\left(1-S_{\alpha}\right) / 3+S_{\alpha} \mathbf{n} \otimes \mathbf{n}$ using the corresponding scalar nematic order parameters $S_{\alpha}$ and obtain the averaged polarizability tensors as

$$
\left\langle\hat{\beta}_{\alpha}\right\rangle=\bar{\beta}_{\alpha} \hat{I}+S_{\alpha} \Delta \beta_{\alpha} \mathbf{n} \otimes \mathbf{n},
$$

where the isotropic polarizabilities $\operatorname{read} \bar{\beta}_{\alpha}=\beta_{\alpha \perp}+$ $\Delta \beta_{\alpha}\left(1-S_{\alpha}\right) / 3$.

Now assuming that moderate anisotropies $\Delta \beta_{\alpha}$ give rise to a relatively small anisotropy of the composite permittivity $\Delta \varepsilon$, one expands Eq. (23) and write

$$
\Delta \varepsilon=\frac{4 \pi}{9}\left(\varepsilon_{\perp}+2\right)^{2}\left(\Delta \beta_{m} \rho_{m} S_{m}+\sum_{n=2}^{\infty}\left\langle\Delta \beta_{n}\right\rangle \rho_{n}\right),
$$

while the isotropic part of the composite permittivity satisfies the generalized Clausius-Mossotti relation

$$
\frac{\varepsilon_{\perp}-1}{\varepsilon_{\perp}+2}=\frac{4 \pi}{3}\left(\beta_{m \perp} \rho_{m}+\beta_{n p} \rho_{n p}+\sum_{n=2}^{\infty} \beta_{n \perp} \rho_{n}\right),
$$


which includes also the contribution from the isotropic non-aggregated NPs.

One notes that Eqs. (25) and (26) are not expected to be quantitatively precise because the neglected correlation corrections may be significant. At the same time, these equations can be used to estimate the dependence of the refractive indices of the nematic composite on the concentration of NPs, their aggregation and ordering provided that the effective polarizability of a NP in the nematic solvent is known.

\section{Low frequency dielectric constant of a strongly polar nematic composite}

Low frequency dielectric constant of the nematic phase composed of strongly polar molecules is mainly determined by the orientational fluctuations of permanent molecular dipoles while the molecular polarizability gives a much smaller contribution. Indeed, the static dielectric constant of a strongly polar nematic can be of the order of 100 while a typical contribution from the molecular polarizability is of the order of 3 [21]. In this case, the macroscopic polarization can be expressed as a sum of averaged molecular dipoles of all components $\alpha$ of the mixture in the unit volume:

$$
\mathbf{P}=\sum_{\alpha} \rho_{\alpha}\left\langle\boldsymbol{\mu}_{\alpha}\right\rangle
$$

where $\boldsymbol{\mu}_{\alpha}$ is the permanent molecule/particle dipole of the component $\alpha$.

In the static case, the average dipole can be expressed as:

$$
\left\langle\boldsymbol{\mu}_{\alpha}\right\rangle=\int \boldsymbol{\mu}_{\alpha} f_{\alpha}(\theta) d \theta
$$

where $f_{\alpha}(\theta)$ is the one-particle distribution function which can be written in the following form in the meanfield approximation

$$
f_{\alpha}(\theta)=Z^{-1} \exp \left[-\beta U_{\mathrm{MF}, \alpha}(\theta)-\left(\boldsymbol{\mu}_{\alpha} \cdot \mathbf{E}\right)\right] .
$$

Here $U_{\mathrm{MF}, \alpha}(\theta)$ is the mean-field potential for the component $\alpha, \theta$ specifies the orientation of the particle/molecule, $\beta=1 / k_{B} T$ and $\mathbf{E}$ is the external electric field.

The mean-field potential can be written in the form:

$$
U_{\mathrm{MF}, \alpha}\left(\theta_{1}\right)=\sum_{\beta} \int V_{\alpha, \beta}\left(\theta_{1}, \theta_{2}\right) f_{\beta}\left(\theta_{2}\right) d \theta_{2},
$$

where $V_{\alpha, \beta}\left(\theta_{1}, \theta_{2}\right)$ is the pair interaction potential between the components $\alpha$ and $\beta$.

Let us now assume that both mesogenic molecules and NPs are uniaxial and their permanent dipoles are parallel to the corresponding long axes. This is also valid for rigid chains of spherical dipolar NPs. In this case, the pair interaction potential $V$ depends on the unit vectors $\mathbf{a}_{1}$ and $\mathbf{a}_{2}$ in the direction of the long axes of the molecules " 1 " and " 2 ", respectively, and on the intermolecular vector $\mathbf{r}_{12}$, i.e. $V(1,2)=V\left(\mathbf{a}_{1}, \mathbf{r}_{12}, \mathbf{a}_{2}\right)$. The pair potential can now be written as a sum of the nonpolar and the polar parts, $V(1,2)=V_{n p}(1,2)+V_{d d}(1,2)$, where the nonpolar potential $V_{n p}(1,2)$ is an even function of $\mathbf{a}_{1}$ and $\mathbf{a}_{2}$ and where the polar potential $V_{d d}(1,2)$ is the electrostatic dipole-dipole interaction potential which can be expressed as:

$$
V_{d d}(1,2)=\boldsymbol{\mu}_{1} \cdot \hat{F}\left(\mathbf{r}_{12}\right) \cdot \boldsymbol{\mu}_{2},
$$

where the dipole-dipole propagator can be written in the form (see Eqs. (4) and (5)):

$$
\hat{F}\left(\mathbf{r}_{12}\right)=\frac{4 \pi}{3} \delta\left(\mathbf{r}_{12}\right)+\Theta\left(r_{12}-D\right)(\hat{I}-3 \mathbf{u} \otimes \mathbf{u}) r_{12}^{-3},
$$

where $\mathbf{u}=\mathbf{r}_{12} / r_{12}$ and where $\Theta\left(r_{12}-D\right)$ is a step function which is equal to unity if $r_{12}>D$ and vanishes otherwise. One notes that the first term in Eq. (32) takes into account a singularity of the dipole-dipole potential at the origin (see a detailed discussion of the averaging of the dipole-dipole potential in Refs. 15 and 22).

Substituting Eq. (32) into Eqs. (31) and (30) and taking into account that the second term in Eq. (32) vanishes after integration over all $\mathbf{u}$, one obtains the final expression for the mean-field potential:

$$
U_{\mathrm{MF}, \alpha}(\theta)=U_{\alpha}^{(0)}(\theta)+\frac{4 \pi}{3}\left(\boldsymbol{\mu}_{\alpha} \cdot \mathbf{P}\right),
$$

Finally this mean-field potential can be substituted into the orientational distribution function (29) and expanding it in powers of the small electric field $\mathbf{E}$ and filedinduced polarization $\mathbf{P}$ one obtains:

$$
f_{\alpha}(\theta) \approx f_{\alpha}^{(0)}\left(1+\frac{4 \pi}{3} \frac{\boldsymbol{\mu}_{\alpha} \cdot \mathbf{P}}{k_{B} T}-\frac{\boldsymbol{\mu}_{\alpha} \cdot \mathbf{E}}{k_{B} T}\right),
$$

where the nonpolar distribution function $f_{\alpha}^{(0)}$ is determined by the nonpolar part $U_{\alpha}^{(0)}(\theta)$ of the mean field potential, that is $f_{0, \alpha}=Z_{0}^{-1} \exp \left[-\beta U_{\alpha}^{(0)}(\theta)\right]$.

Substituting Eq. (34) into Eqs. (28) and (27) one obtains the following linear equation for the macroscopic polarization $\mathbf{P}$ :

$$
P_{i}=\sum_{\alpha} \frac{\rho_{\alpha}}{k_{B} T}\left\langle\mu_{\alpha, i} \mu_{\alpha, j}\right\rangle_{0}\left(\frac{4 \pi}{3} P_{j}+E_{j}\right),
$$

where the averaging $\left\langle\mu_{\alpha, i} \mu_{\alpha, j}\right\rangle_{0}$ is performed with the nonpolar orientational distribution function $f_{\alpha}^{(0)}$. As a result, one obtains the following expression for the dielectric polarizability tensor $\hat{\chi}$ :

$$
\hat{\chi}=\frac{\hat{\chi}_{0}}{1-\frac{4 \pi}{3} \hat{\chi}_{0}}
$$


where

$$
\hat{\chi}_{0}=\sum_{\alpha} \frac{\rho_{\alpha}}{k_{B} T}\left\langle\boldsymbol{\mu}_{\alpha} \otimes \boldsymbol{\mu}_{\alpha}\right\rangle_{0}
$$

Taking into account that the dipole $\boldsymbol{\mu}_{\alpha}$ is parallel to the long axis a of the corresponding molecule one obtains:

$$
\begin{aligned}
\hat{\chi}_{0}=\sum_{\alpha} \frac{\rho_{\alpha} \mu_{\alpha}^{2}}{k_{B} T}\langle\mathbf{a} \otimes \mathbf{a}\rangle_{\mathbf{0}}= \\
\sum_{\alpha} \frac{\rho_{\alpha} \mu_{\alpha}^{2}}{k_{B} T}\left[S_{\alpha}(\mathbf{n} \otimes \mathbf{n}-\hat{I} / 3)+\hat{I} / 3\right] .
\end{aligned}
$$

Here $S_{\alpha}$ is the nematic order parameter of the compound $\alpha$ in the mixture.

\section{CONTRIBUTION OF NANOPARTICLE CHAINS TO THE DIELECTRIC ANISOTROPY OF A NEMATIC COMPOSITE}

One notes that the dielectric properties of the composite essentially depend on the densities $\rho_{m}$ of NP chains of length $m$. The latter can be evaluated using the existing theory of chain formation in the system of polar spheres presented, for example, in Ref. 15. According to this theory, the distribution of chain lengths is expressed as:

$$
\phi_{m}=v \rho_{m}=e^{m\left(U_{0}+\nu\right)} e^{-U_{0}},
$$

where $\phi_{m}$ is the volume fraction of chains of length $m, v$ is the NP volume and $U_{0}$ is the contact energy determined by the dipole-dipole interaction between NPs:

$$
U_{0}=\ln \left(\frac{\pi \sigma^{3} e^{2 \lambda}}{18 v \lambda^{3}}\right) .
$$

Here $\lambda=\mu^{2} / k_{B} T \sigma^{3}$ has to be sufficiently larger than unity, $\sigma$ is the NP diameter, and the NP volume $v$ has been introduced for dimensional correctness.

In Eq. (39), $\nu$ is the Lagrange multiplier (chemical potential) which is determined from the conservation rule for NPs:

$$
\rho=\sum_{m=1}^{\infty} m \rho_{m},
$$

where $\rho$ is the molar fraction of all NPs which typically is controlled in experiments.

Substituting Eq. (39) into Eq. (41) and performing the summation one obtains:

$$
\rho=v^{-1} \frac{e^{\nu}}{\left(1-e^{U_{0}+\nu}\right)^{2}} .
$$

Accordingly,

$$
1-e^{U_{0}+\nu}=\frac{-1+\sqrt{1+4 \eta}}{2 \eta}
$$

where $\eta=v \rho e^{U_{0}}$. Thus the value of the chemical potential $\nu$ is mainly determined by the order of $\eta$.

Finally, one can readily obtain the following expression for the number density of chains of length $m$ :

$$
\rho_{m}=v^{-1} e^{-U_{0}}\left(1-\frac{-1+\sqrt{1+4 \eta}}{2 \eta}\right)^{m} .
$$

In principle, the order parameters $S_{m}$ are different for different $m$ but this difference is not huge. Thus for a crude estimate, we can assume that $S_{m}=S$ where $S$ is the order parameter of the host nematic.

\section{A. High-frequency dielectric anisotropy}

Generally, the NP contribution to the composite permittivity (25) and (26) is twofold: both aggregated and non-aggregated NPs affect $\varepsilon_{\perp}$ while only those NPs which are aggregated into chains contribute to $\Delta \varepsilon$.

To sum over chains of different lengths in Eq. (25) one needs to know the quantity $\left\langle\Delta \beta_{n}\right\rangle$ which can be evaluated as the average dielectric anisotropy of a chain of $n$ spheres (with the permittivity $\varepsilon_{n p}$ ) immersed into a medium with the permittivity $\varepsilon_{\perp}$. Although the exact solution of such problems can be performed only numerically, upon assuming a few realistic approximations one can obtain useful analytical estimates [23]. Thus taking into account the strongest dipole interactions between NPs and restricting to the nearest-neighbor contributions (already the next-nearest ones are at least eight times smaller) and introducing the single NP dielectric polarizability $\beta_{1}=1 / 8 \sigma^{3}\left(\varepsilon_{n p}-\varepsilon_{\perp}\right) /\left(\varepsilon_{n p}+2 \varepsilon_{\perp}\right)$ one can express the dipole moment of an $l$-th NP in the chain of the total length $m$ as

$$
\mathbf{p}_{l}=\beta_{1} \mathbf{E}+\beta_{1} \hat{T}_{l, l-1} \mathbf{p}_{l-1}+\beta_{1} \hat{T}_{l, l+1} \mathbf{p}_{l+1},
$$

where

$$
\hat{T}_{l, l \pm 1}=\left(3 \mathbf{u}_{l, l \pm 1} \otimes \mathbf{u}_{l, l \pm 1}-1\right) \sigma^{-3},
$$

is the non-singular part of the dipole-dipole propagator, $\mathbf{u}_{l, l \pm 1}$ are the unit vectors between the centers of the adjacent NPs and the following natural condition is satisfied $\hat{T}_{1,0}=\hat{T}_{m, m+1}=0$ at the chain ends.

As shown below, the effect of chain formation on highfrequency permittivity is rather moderate, and one can solve the system (45) by iterations. While in the zeroth order (neglecting the NP interactions) one obtains merely $\mathbf{p}_{l}=\beta_{1} \mathbf{E}$ and the chain remains dielectrically isotropic, the next iteration yields:

$$
\mathbf{p}_{l}=\beta_{1}\left(1+\beta_{1} \hat{T}_{l, l-1}+\beta_{1} \hat{T}_{l, l+1}\right) \mathbf{E} .
$$

Since the average chain direction is controlled by the overall composite nematic director $\mathbf{n}$, the averaged nearest-neighbor propagator reads

$$
\left\langle\hat{T}_{l, l \pm 1}\right\rangle=S(3 \mathbf{n} \otimes \mathbf{n}-1) \sigma^{-3},
$$




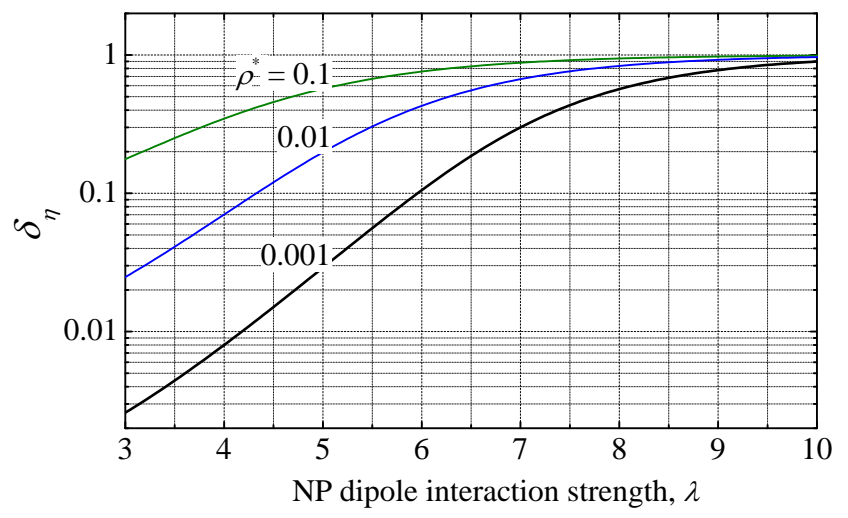

FIG. 1. The effect of chain formation on the birefringence of the nematic composite: dependences of the factor $\delta_{\eta}$ given by Eq. (54) on the NP coupling strength for the NP density $\rho^{*}=0.1,0.01$ and 0.001 as indicated on the lines.

where we have again assumed that all the scalar nematic order parameters in the composite are equal.

Evaluating the average chain dipole moment as $\left\langle\mathbf{P}_{m}\right\rangle=\sum_{l=1}^{m}\left\langle\mathbf{p}_{l}\right\rangle$ one obtains the following expression for the overall average chain polarizability tensor

$$
\left\langle\hat{\beta}_{m}\right\rangle=m \beta_{1} \hat{I}+\frac{2}{\sigma^{3}}(m-1) \beta_{1}^{2} S(3 \mathbf{n} \otimes \mathbf{n}-1) .
$$

The anisotropy of this polarizability is given by:

$$
\left\langle\Delta \beta_{m}\right\rangle=\frac{6}{\sigma^{3}}(m-1) \beta_{1}^{2} S .
$$

Accordingly, the chain contribution to the composite permittivity anisotropy (25) is given by

$$
\Delta \varepsilon_{\mathrm{ch}}=\frac{\pi}{24}\left[\frac{\left(\varepsilon_{\perp}+2\right)\left(\varepsilon_{n p}-\varepsilon_{\perp}\right)}{\varepsilon_{n p}+2 \varepsilon_{\perp}}\right]^{2} S \sigma^{3} \sum_{m=2}^{\infty}(m-1) \rho_{m} .
$$

Substituting the number densities (44) an using the summation rule

$$
\sum_{m=2}^{\infty}(m-1) x^{m}=\frac{x^{2}}{(1-x)^{2}}
$$

one can express the dielectric anisotropy in terms of the dimensionless NP density $\rho^{*}=\rho \sigma^{3}$ and the parameter $\lambda$ :

$$
\Delta \varepsilon_{\mathrm{ch}}=\frac{\pi}{24}\left[\frac{\left(\varepsilon_{\perp}+2\right)\left(\varepsilon_{n p}-\varepsilon_{\perp}\right)}{\varepsilon_{n p}+2 \varepsilon_{\perp}}\right]^{2} S \rho^{*} \delta_{\eta}
$$

where the function

$$
\delta_{\eta}=2+\frac{1}{\eta}-\frac{4 \eta}{(\sqrt{1+4 \eta}-1)^{2}}
$$

effectively describes the dependence on the NP chain formation as $\eta$ is also expressed in terms of the nondimensional parameters as $\eta=\pi \rho^{*} e^{2 \lambda} /\left(18 \lambda^{3}\right)$.

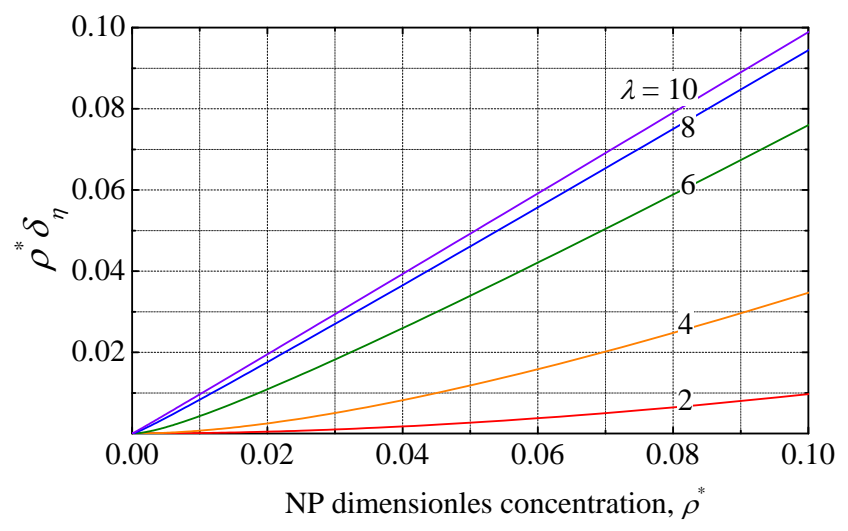

FIG. 2. The effect of chain formation on the birefringence of the composite: dependences of the factor $\rho^{*} \delta_{\eta}$ in Eq. (53) on the NP concentration for the coupling strength $\lambda$ varying from 2 to 10 as indicated on the lines.

Representative profiles of the factor $\delta_{\eta}$ as functions of the NP coupling strength, which controls the chain formation, are presented in Fig. 1. One notes that for weaker coupling this factor is very small, as most of the NPs remain single here and do not contribute to the anisotropy. For stronger coupling, the average chain length increases which leads to a pronounced increase of the anisotropy. The saturation at $\delta_{\alpha} \approx 1$ for strongly interacting NPs means that in this limit practically all NPs belong to long chains and contribute equally to the anisotropy. Evidently, for higher total NP concentrations this saturation occurs at smaller $\lambda$.

The chain contribution to the composite birefringence as a function of the NP concentration is illustrated by Fig. 2 for different values of the dipole-dipole interaction strength. One notes that it is practically a linear function when the NP coupling is strong enough, i.e., when all the NPs are aggregated in long chains.

Generally, the high-frequency anisotropy is weak as the factor $\delta_{\alpha}<1$ is multiplied in Eq. (53) by a number of other small factors. Thus for the dielectric NPs with $\varepsilon_{n p}$ of the same sign and order of magnitude as $\varepsilon_{\perp}$, the factors in the square brackets are of the order of unity, while $S<1$ and $\rho^{*} \ll 1$. On the other hand, the variation of $\delta_{\alpha}$ by three orders of magnitude for low $\rho^{*}=0.001$ in Fig. 1 suggests that this anisotropy can be employed as a sensitive tool for quantitative assessment of the NP chain formation in nematic composites.

\section{B. Low-frequency dielectric anisotropy}

Let us consider the nematic composite in which the permanent dipoles of NPs are sufficiently large and larger than those of the mesogenic molecules. Then the main contribution to the low frequency dielectric constant of the nano-composite stems from the NPs and their chains 

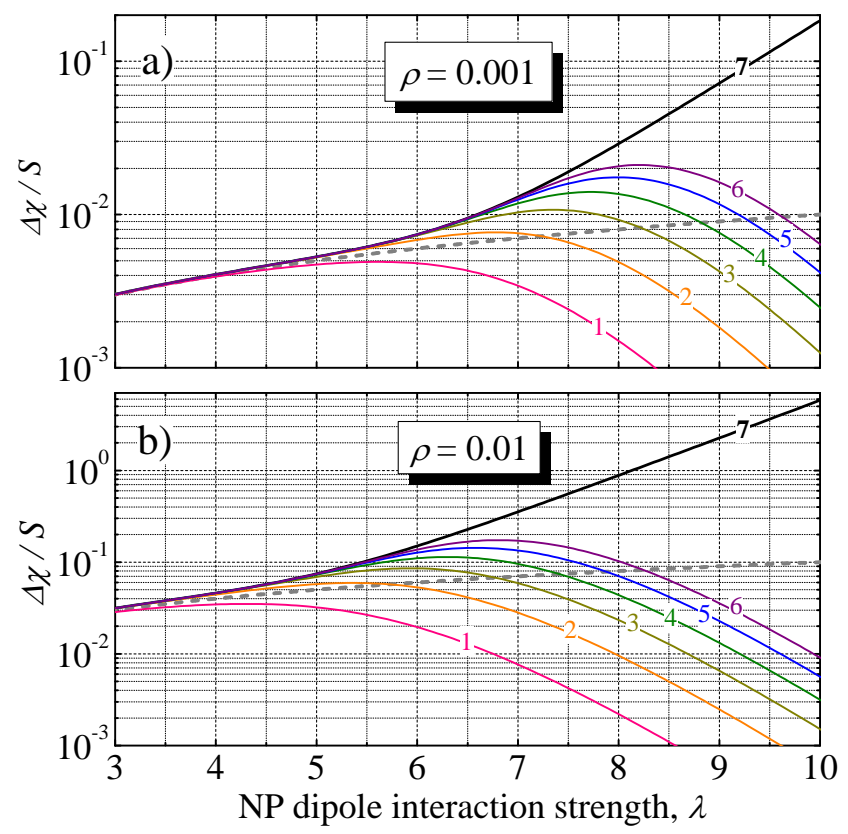

FIG. 3. Anisotropy of the low-frequency composite dielectric susceptibility as a function of NP coupling strength for NP densities $\rho^{*}=0.001$ (a) and $\rho^{*}=0.01$ (b). Solid lines $1-$ 6 depict results of partial summation in Eq. (56) neglecting chains with $m$ higher than $1-6$ correspondingly. Solid line 7 represents the dependence (58), and the dashed line shows the anisotropy in the absence of chain formation.

and can be written using Eq. (38) as:

$$
\begin{aligned}
& \hat{\varepsilon} \approx 1+4 \pi \hat{\chi}_{0}= \\
& \quad 1+4 \pi \sum_{m=1} \frac{\rho_{m} \mu_{m}^{2}}{k_{B} T}\left[S_{m}(\mathbf{n} \otimes \mathbf{n}-\hat{I} / 3)+\hat{I} / 3\right]
\end{aligned}
$$

where $\mu_{m}$ is the total dipole of the chain of length $m, \rho_{m}$ is the number density of chains of length $m$ and $S_{m}$ is the corresponding nematic order parameter.

One may assume that for short rigid chains of polar NPs, the total dipole $\mu_{m}=m \mu$ where $\mu$ is the permanent dipole of a single NP. This assumption is obviously not valid for long flexible chains. However, the concentration of such chains is exponentially small and we will see below that for realistic values of the NP dipole only short chains $(m=1-4)$ make a significant contribution to the dielectric constant of the composite. In this approximation Eq. (55) yields the dielectric susceptibility anisotropy:

$$
\Delta \chi=\frac{\mu^{2}}{k_{B} T} \sum_{m=1} m^{2} \rho_{m} S_{m}
$$

Neglecting the effect of weak external electric field on the chain formation statistics, one can substitute here the number densities (44), set for simplicity $S_{m}=S$, and perform the summation over chains of all lengths

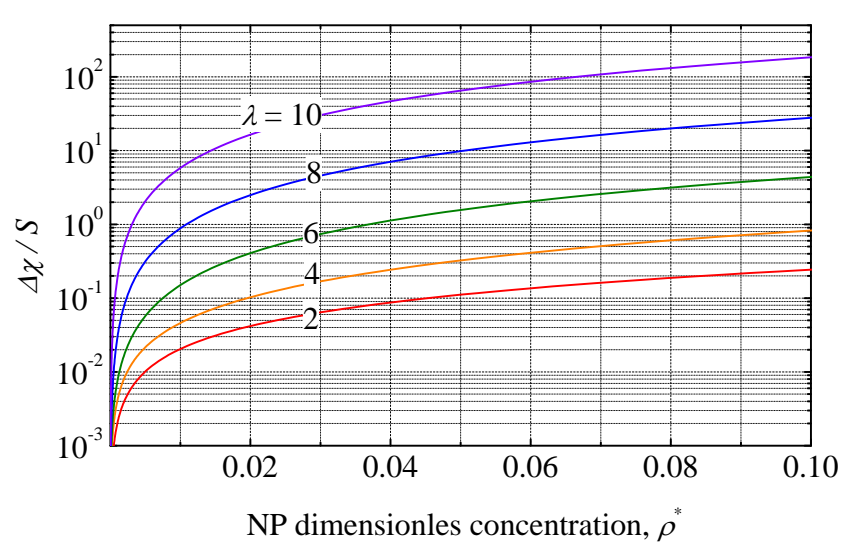

FIG. 4. Anisotropy of the low-frequency composite dielectric susceptibility as a function of the NP concentration for the NP interaction strength $\lambda$ varying from 2 to 10 as indicated on the lines.

using the summation rule

$$
\sum_{m=1}^{\infty} m^{2} x^{m}=\frac{x(1+x)}{(1-x)^{3}}
$$

Then the low-frequency dielectric anisotropy (56) can be expressed explicitly in terms of $\rho^{*}$ and $\lambda$ :

$$
\Delta \chi=4 \rho^{*} \lambda S \frac{4 \eta^{2}+5 \eta+1-(3 \eta+1) \sqrt{1+4 \eta}}{(-1+\sqrt{1+4 \eta})^{3}} .
$$

In Fig. 3, the dielectric anisotropy given by Eq. (58) is presented for different NP molar fractions as a function of the parameter $\lambda$ which describes the strength of the dipole-dipole interaction between NPs. For comparison we also present the corresponding variation $\Delta \tilde{\chi}=\lambda S \rho^{*}$ of the dielectric anisotropy of the composite without any chains, as well as the results of the partial summation in Eq. (56) which show the relative scale of contributions from chains of different lengths. One can see that the chain formation can modify the dielectric properties by orders of magnitude when the NP interaction (determined by the permanent dipole) is sufficiently strong. At the same time, for weak interaction, the effect of chains is practically negligible and the NPs respond to the electric field independently. For moderate interactions, there exists a noticeable area of $\lambda$, where the formation of short chains (dimers and trimers) contributes to $\Delta \chi$ considerably, while the effect of longer chains is practically absent.

One can readily see in Fig. 3 that a contribution from monomers and dimers (similar to that from monomers and $n$-mers for $n=3,4,5)$ first increases with the increasing dipolar strength $\lambda$, then reaches a maximum and finally begins to decrease. The decreasing stage corresponds to the range of $\lambda$ which correspond to the formation of longer chains which make a predominant contribution to the dielectric anisotropy. In this range, the contribution from dimers, trimers etc. decreases due to a decrease of the corresponding number densities. The 
increasing stage corresponds to the range of smaller $\lambda$ where the corresponding short chains make a predominant contribution.

Finally, the variation of the dielectric anisotropy as a function of the NP concentration for different values of the dipole-dipole interaction strength is presented in Fig. 4. Evidently, the increase of the NP concentration by an order of magnitude results in the increase of the dielectric anisotropy by several orders of magnitude depending on the value of the parameter $\lambda$. Thus one can readily see (compare also with Figs. 3a and 3b) that the experimentally observed increase of the dielectric constant $[9,13]$ at very low NP number density $\rho=10^{-2}-10^{-3}$ can be explained by the effect of chain formation only if the dipole-dipole interaction strength is sufficiently high which is the case for ferroelectric NPs with large spontaneous polarization.

\section{DISCUSSION}

In this paper we have presented a general statistical theory of the dielectric susceptibility of nematic liquid crystal mixtures, including nematics doped with polar NPs, which in principle enables one to take into account intermolecular and interparticle correlations. At this stage, a general theory can only be developed for the limiting cases of high and low frequency. The general theory has been used to obtain an approximate explicit expression for the sum of contributions from NP chains of all lengths to the birefringence and to the anisotropy of the static dielectric constant and to study the dependence of the dielectric anisotropy on the NP concentration and the dipole-dipole interaction strength which is mainly determined by the value of the NP permanent dipole.

Strongly polar NPs are expected to form chains or, at least, dimers and trimers, which can make a significant contribution to the dielectric constant of the nematic composite. In fact a contribution from chains of strongly polar NPs can be orders of magnitude larger than that of single NPs. In the case of ferroelectric NPs with large spontaneous polarization $[9,13,18,24]$ the dipole-dipole interaction at the contact distance between NPs is large enough and this enables one to explain why doping of a nematic LC with a very low concentration of NPs (with number density of the order of $10^{-3}$ ) may result in a substantial increase of the dielectric anisotropy comparable to the anisotropy of the nematic host itself $[9,13]$. In the case of moderate dipole-dipole interaction between NPs, the dielectric anisotropy is mainly determined by short chains including dimers, trimers, etc., which still make a much larger contribution than individual NPs. The dependence of the dielectric anisotropy on the concentration of NPs has also been evaluated numerically.

The general theory developed in this paper has also been used to evaluate a contribution of long chains to the birefringence of the composite nematic phase. The birefringence has been evaluated as a function of the NP concentration and the dipole-dipole interaction strength. As shown in Section III, the behavior of the birefringence at low non dimensional molar fraction of NPs (e.g. at $\rho^{*}=0.001$ ) can be used for a quantitative assessment of the chain formation in nematic composites.

Finally, one notes that the formation of dimers of magnetic dipolar spherical NPs in an isotropic fluid at extremely low concentration of NPs accounts for the experimentally observed birefringence induced by the external magnetic field [25]. In such a fluid, the macroscopic magnetic anisotropy is determined by the orientational ordering of dimers of magnetic NPs induced by the external field, and theoretical estimates of dimer concentration can be used to explain the experimentally observed dependence of the birefringence on the external magnetic field [25].

\section{ACKNOWLEDGMENTS}

The authors are grateful to J. Goodby, Yu. Reznikov and R.V. Talroze for interesting discussions. MVG acknowledges the support from the Russian Foundation for Basic Research (projects No.13-03-00579 and No.14-0390016).
[1] H. Qi, B. Kinkead, T. Hegmann, Proc. SPIE 2008, 6911, 691106 (2008).

[2] H. Qi and T. Hegmann, J. Mater. Chem. 16, 4197 (2006).

[3] Y. Shiraishi, N. Toshima, K. Maeds, H. Yoshikawa, J. Xu, and S. Kobayashi: Appl. Phys. Lett. 812845 (2002).

[4] S.Kobayashi and N.Toshima, Information Display, 23, 26 (2007).

[5] H. Yoshida, K. Kawamoto, H. Kubo, T. Tsuda, A. Fujii, S. Kuwabata, M. Ozaki, Advanced Materials 22, 622 (2010).

[6] S. Kaur, S.P. Singh, A.M. Biradar, A. Choudhary, and K. Sreeniva, Appl. Phys. Lett. 91, 023120 (2007).
[7] A. Kumar, J. Prakash, D.S. Mehta, A.M. Biradar, and W. Haase Appl. Phys. Lett. 95, 023117, (2009).

[8] O. Buchnev, A. Dyadyusha, M. Kaczmarek, V. Reshetnyak, Yu. Reznikov, J. Opt. Soc. Am. B 24, 1512 (2007).

[9] Yu. Reznikov, O. Buchnev, O. Tereshchenko, V. Reshetnyak, A. Glushchenko, and J. West, Appl. Phys. Lett. 82, 1917 (2003)

[10] L.M. Lopatina and J.V. Selinger, Phys. Rev. Lett. 102, 197802 (2009).

[11] H. Yoshida et al Appl. Phys. Expr. 2, 121501 (2009).

[12] S. Wiersma, Nat. Phys. 4, 359 (2008).

[13] E. Ouskova, O. Bunchev, V. Reshetnyak and Yu. Reznikov, Liq.Cryst. 30, 1235 (2003). 
[14] R. Basu and G.S. Iannacchione, Phys. Rev. E 80, 010701(R) (2009).

[15] M.A. Osipov, P.I.C. Teixeira and M.M. Telo da Gama, Phys. Rev. E 54, 2597 (1996).

[16] M.V. Gorkunov and M.A. Osipov, Soft Matter 7, 4348 (2011).

[17] Y.L. Raikher, V.I. Stepanov and A.N. Zakhlevnykh, Soft Matter 9, 177 (2013).

[18] L.M. Lopatina and J.V. Selinger, Phys. Rev. E 84, 041703 (2011).

[19] M.A. Osipov and M. Gorkunov, ChemPhys.Chem. 15, 1496 (2014).
[20] D. Bedeaux and P. Mazur, Physica 67, 23 (1973).

[21] W.H. de Jeu and P. Bordewijk, J. Chem. Phys. 68, 10915 (1978).

[22] Osipov, M. , Pajak, G. Eur. Phys. J. E 37, 79 (2014).

[23] M. Quinten, Optical Properties of Nanoparticle Systems, Mie and Beyond, Wiley-VCH (2011).

[24] F. Li, O. Buchnev, C.I. Cheon, A. Glushchenko, V. Reshetnyak, Yu. Reznikov, T.J. Sluckin, and J.L. West, Phys. Rev. Lett. 97, 147801 (2006); 99, 219901 (E) (2007).

[25] J. Szczytko, N. Vaupotic, M.A. Osipov, K. Madrak, and E. Gorecka, Phys. Rev. E. 87, 062322 (2013). 